\title{
Five-year patency for the no-touch saphenous vein and the left internal thoracic artery in on- and off-pump coronary artery bypass grafting
}

\author{
Mikael Arbeus ${ }^{1}$, Domingos Souza ${ }^{1}$, Håkan Geijer ${ }^{1}$, Mats Lidén ${ }^{1}$, Bruno Pinheiro ${ }^{2}$, Lennart \\ Bodin $^{3}$, and Ninos Samano ${ }^{4}$ \\ ${ }^{1}$ Örebro universitet \\ ${ }^{2}$ Hospital do Coracao Anis Rassi Ltda \\ ${ }^{3}$ Karolinska Institute \\ ${ }^{4}$ Uppsala University
}

June 3, 2021

\begin{abstract}
Background Randomised trials show high long-term patency for no-touch saphenous vein grafts in coronary artery bypass grafting. The patency rate in off-pump coronary bypass surgery for these grafts has not been investigated. Our centre participated in the CORONARY randomized trial, NCT00463294. This is a sub-study aimed to assess the patency of no-touch saphenous veins in on- versus off-pump coronary bypass surgery at five-year follow-up. Methods Fifty-six patients were included. Forty of 49 patients, alive at five years, participated in this follow-up. There were 21 and 19 patients in the on- and off-pump groups respectively. No-touch saphenous veins were used to bypass all targets and in some cases the left anterior descending artery. Graft patency according to distal anastomosis was evaluated with computed tomography angiography. Results The five-year patency rate was $123 / 139(88.5 \%)$. The patency for the no-touch vein grafts was $57 / 64(89.1 \%)$ in the on-pump vs $37 / 45(82.2 \%)$ in the off-pump group. All left internal thoracic arteries except for one, 29/30 (96.6\%), were patent. All vein grafts used to bypass the left anterior descending and the diagonal arteries were patent $32 / 32$. The lowest patency rate for the saphenous veins was to the right coronary territory, particularly in off-pump surgery $(80.0 \%$ vs $62.5 \%$ for the on- respective off-pump groups). Conclusions Comparable five-year patency for the no-touch saphenous veins and the left internal thoracic arteries to the left anterior descending territory in both on- and off-pump coronary artery bypass grafting. Graft patency in off-pump CABG is lower to the right coronary artery.
\end{abstract}

Five-year patency for the no-touch saphenous vein and the left internal thoracic artery in onand off-pump coronary artery bypass grafting

Mikael Arbeus ${ }^{1} \mathrm{MD}$, DomingosSouza ${ }^{1} \mathrm{PhD}$, HåkanGeijer ${ }^{2} \mathrm{PhD}$, MatsLidén ${ }^{2} \mathrm{PhD}$, BrunoPinheiro ${ }^{3} \mathrm{MSc}$, LennartBodin ${ }^{4} \mathrm{PhD}$, NinosSamano ${ }^{5} \mathrm{PhD}$

${ }^{1}$ Department of Cardiothoracic and Vascular Surgery, Faculty of Medicine and Health, Örebro University, Örebro, Sweden, ${ }^{2}$ Department of Radiology, Faculty of Medicine and Health, Örebro University, Örebro, Sweden, ${ }^{3}$ Department of Cardiovascular Surgery, Hospital do Coracao Anis Rassi, Goiania, Brazil, ${ }^{4}$ Intervention and Implementation Research, Institute of Environmental Medicine, Karolinska Institute, Stockholm, Sweden, ${ }^{5}$ Department of Cardiothoracic Surgery, Uppsala University Hospital and Department of Surgical Sciences, Thoracic Anaesthesiology and Intensive Care, Uppsala University, Uppsala, Sweden.

Funding: Funded by the Örebro County Council through the Regional Research Board and Nyckelfonden (grant \# OLL-689991). 
Meeting presentation: EACTS annual meeting in Vienna, Austria, 7-11 October 2017.

Disclosures: There are no financial conflicts or other conflicts of interest related to the material of the present study.

Registration: ClinicalTrials.gov NCT03447197

Corresponding author: Dr. Ninos Samano

Address: Department of Cardiothoracic Surgery, Uppsala University Hospital, SE-751 85, Uppsala, Sweden.

Office: +46722064392

$+46739544081$

E-mail:ninos.samano@gmail.com

Word count: 4458

References: 20

Abstract

Background

Randomised trials show high long-term patency for no-touch saphenous vein grafts in coronary artery bypass grafting. The patency rate in off-pump coronary bypass surgery for these grafts has not been investigated. Our centre participated in the CORONARY randomized trial, NCT00463294. This is a study aimed to assess the patency of no-touch saphenous veins in on- versus off-pump coronary bypass surgery at five-year follow-up.

\section{Methods}

Fifty-six patients were included. Forty of 49 patients, alive at five years, participated in this follow-up. There were 21 and 19 patients in the on- and off-pump groups respectively. No-touch saphenous veins were used to bypass all targets and in some cases the left anterior descending artery. Graft patency according to distal anastomosis was evaluated with computed tomography angiography.

\section{Results}

The five-year patency rate was 123/139 (88.5\%). The patency for the no-touch vein grafts was 57/64 (89.1\%) in the on-pump vs $37 / 45(82.2 \%)$ in the off-pump group. All left internal thoracic arteries except for one, 29/30 (96.6\%), were patent. All vein grafts used to bypass the left anterior descending and the diagonal arteries were patent $32 / 32$. The lowest patency rate for the saphenous veins was to the right coronary territory, particularly in off-pump surgery ( $80.0 \%$ vs $62.5 \%$ for the on- respective off-pump groups).

\section{Conclusions}

Comparable five-year patency for the no-touch saphenous veins and the left internal thoracic arteries to the left anterior descending territory in both on- and off-pump coronary artery bypass grafting. Graft patency in off-pump CABG is lower to the right coronary artery.

Keywords : Coronary artery bypass grafting, no-touch, off-pump, patency, saphenous veins.

\section{Abbreviations}

CABG coronary artery bypass grafting

CI confidence interval

CPB cardiopulmonary bypass

Cx circumflex 
D diagonal

LAD left anterior descending

LITA left internal thoracic artery

NT no-touch

NT SVG no-touch saphenous vein graft

NYHA New York Heart Association

PCI percutaneous coronary intervention

PDA posterior descending artery

RCA right coronary artery

SV saphenous vein

SVG saphenous vein graft

\section{Introduction}

The saphenous vein (SV)continues to be a very important conduit in coronary artery bypass grafting (CABG) ${ }^{1}$. Therefore, every effort should be made to improve the short and long-term patency of SV grafts (SVGs). The no-touch (NT) SVG harvesting technique was introduced in the 1990s. The NT SVG is harvested together with its fat pedicle preventing spasm and the need for manual dilatation ${ }^{2}$. A randomized study comparing the NT to the conventional vein harvesting technique, showed significantly higher patency for NT SVGs comparable to that of the left internal thoracic artery (LITA) 16 years postoperatively ${ }^{3-5}$. Coronary artery bypass grafting is commonly performed with the support of cardiopulmonary bypass (CPB), so called on-pump procedure. Perioperative mortality is about $2 \%$ and the rate of myocardial infarction, stroke or renal failure requiring dialysis is $5-7 \%{ }^{6}$. The technique of performing CABG without CPB, off-pump surgery, was developed to reduce these perioperative complications ${ }^{7}$.

The CABG Off or On-pump Revascularization study (CORONARY trial, NCT00463294) is a large international multicenter randomized controlled trial. It was designed to overcome some of the limitations of prior trials ${ }^{8}$. Our department was one of the including centers. The 30-days, one year and five-year results have been published ${ }^{6,9}$. Graft patency was not evaluated in this study despite being a major determinant of clinical prognosis, measured by reoperation rates and long-term survival ${ }^{10}$. Therefore, the aim of this sub-study is to assess graft patency in on- vs off-pump CABG in the cohort of patients operated at our department, especially considering that all vein grafts were harvested with the NT technique. Graft patency according to the different target coronary arteries was also studied.

\section{Material and Methods}

At our center, 56 patients were included in the CORONARY trial according to the inclusion criteria of the original protocol ${ }^{8}$. Forty-nine patients were alive at the five-year follow-up, see figure 1 . In on-pump CABG, CPB was instituted without active cooling and cold $\left(4^{\circ} \mathrm{C}\right)$ anterograde blood cardioplegia solution, containing $20 \mathrm{mmol} / \mathrm{l}$ potassium, was used for myocardial protection. In the off-pump group, an Octopus Evolution Stabilizer (Medtronic, Minneapolis, USA) and the Heartstring III (Maquet, Karlsruhe, Germany) $4.3 \mathrm{~mm}$ device for proximal anastomosis were used. In this group, the proximal anastomoses were performed before grafting of the distal targets. No conversion between the techniques was needed. Two surgeons who fulfilled the expertise criteria performed the procedures ${ }^{8}$. The SV was always harvested according to the NT technique ${ }^{2}$. All patients scheduled to undergo the five-year clinical follow-up were asked to undergo a computed tomography angiography (CTA) to evaluate graft patency. The study was approved by the Regional Ethical Review Board. All the patients were included at follow-up after informed consent. Baseline data was retrieved from our local registry. 


\section{Statistical methods}

Statistical analysis was performed with a multi-level logistic regression model for patency when data for patency was given in 2-by-2 tables stratified by type of technique (on-pump or off-pump) and harvesting technique. The main predictor variable for patency was the two different techniques, on- or off-pump. Most patients had more than one graft, with a maximum number of three grafts per patient. Multiple grafts per patient might introduce dependencies between the observations. We corrected for clustered observations in the calculation of confidence intervals (CIs), with each patient forming a separate cluster.

With the aim of investigating a possible equivalence between on- and off-pump, the graphical approach recommended by Fleming ${ }^{11}$ and by Blackwelder ${ }^{12}$ was used. An essential point in this approach is to define, based on experience, limits of what can be regarded as equivalent outcomes with respect to patency. Therefore, no formal statistical hypothesis testing was conducted, and instead results are shown in graphics with CIs for the differences in patency rates between the two techniques. Through these CIs, possible equivalences between on- and off-pump can be visualised. When the CI does not cover the point of no difference in patency between the methods (the '0-point'), equivalence between the techniques is rejected. If, on the other hand, the CI covers the '0-point' and in addition the CI does not stretch outside the equivalence limits there is an evidence for equivalence. Finally, if the '0-point' is covered by the CI but at least one of the two limits of CI stretches outside the equivalence limits the result is inclusive, possibly due to lack of power. Computations were performed with STATA version 15 (www.stata.com).

\section{Radiological methods}

This was performed using a Somatom Flash dual-source CT scanner (Siemens, Erlangen, Germany). All subjects received $0.25 \mathrm{mg}$ of Nitroglycerin sublingually. Those with a heart frequency $>70$ beats/minute and no contraindications were also given up to $10 \mathrm{mg}$ of Metoprolol intravenously before the examination. Contrast media (60-70 ml Iomeron $400 \mathrm{mg} / \mathrm{ml}$, Bracco, Milan, Italy) was administered with a pressure injector at a flow rate of $6 \mathrm{ml} / \mathrm{second}$, followed by a bolus of $60 \mathrm{ml}$ saline. Scanning started at the left subclavian artery and ended at the base of the heart. The images were reviewed at a Siemens SyngoVia workstation. All images were independently reviewed by two thoracic radiologists who were blinded to group assignment. Disagreements were resolved by consensus. A graft was judged as occluded when the graft was not opacified by contrast media. A graft stenosis was judged as significant when the narrowing of the lumen diameter was $>50 \%$ relative to the adjacent parts of the vessel.

\section{Results}

Fifty-six patients were included in the original study at our center. Thirty vs 26 patients for the on- respective off-pump groups. All patients were alive 30 days postoperatively. One patient in each group needed early reoperation for bleeding. Two patients developed sternal wound infections, 2/56 (3.6\%) and three patients had leg wound infections, 3/56 (5.3\%). One patient in the on-pump group needed repeat revascularization with percutaneous coronary intervention (PCI) and one patient in the off-pump group underwent redo surgery due to new coronary lesions and mitral regurgitation. Five patients in the on-pump group and two patients in the off-pump group had died, 7/56 (12.5\%). Five out of seven patients died from non-cardiac causes.

The five-year follow-up included 49 patients with 25 vs 24 patients in the on- and off-pump groups respectively. All patients underwent a clinical evaluation according to protocol. Five patients were excluded from CTA due to renal failure and two patients in each group refused to participate in this sub-study. Forty patients underwent CTA with 139 distal anastomoses analyzed, see figure 1. Patient characteristics at five years are reported, see table 1. Similar mean age, gender distribution and most risk factors between the groups. All patients were on anti-platelet therapy. Some numerical differences between the two groups, for example more patients in NYHA class I in the on-pump group. No patient reported pain from the SV harvesting site and only one patient reported numbness. Small coronary targets, [?] $1 \mathrm{~mm}$ in diameter, were more frequent in the right coronary territory, 10/41 (24.7\%). Six patients received a NT SVG to left anterior descending (LAD) artery due to either borderline stenosis or surgical injury to the LITA. In four patients the NT SVG was used to substitute the LITA in patients with multiple co-morbidities. The mean grafting rate per patient 
was 3.8 and 3.1 distal anastomoses in the on- and off-pump groups respectively.

The overall five-year patency rate according to the number of distal anastomoses was $123 / 139,(88.5 \%)$ of which $73 / 80(91.3 \%)$ were in the on-pump group and 50/59 (84.7\%) in the off-pump group. The patency rate was higher for the grafts used to the LAD territory than for those grafts used to both the circumflex $(\mathrm{Cx})$ and the right coronary territories, independent of the surgical method used, see figure 2 . The total patency rate for LITA was 29/30 (96.7\%) and for the NT SVGs 94/109 $(86.2 \%)$. The patency rate of LITA was similar between the groups, on-pump 16/16 (100\%) and off-pump 13/14 (92.8\%). The patency rate for NT SVGs was also similar between both surgical procedures, 57/64 (89.1\%) in the on-pump group and 37/45 $(82.2 \%)$ in the off-pump group. However, all NT SVGs that were used to bypass the LAD and the diagonal (D) branches were patent, 20/20 grafts in the on-pump group and12/12 grafts in the off-pump group. The lowest patency for the NT SVG was to the right coronary territory, particularly in off-pump surgery, 16/20 $(80.0 \%)$ and $10 / 16(62.5 \%)$ for the on- and off-pump groups respectively, see table 2.

Analyses of a possible superiority in patency for the two surgical procedures stratified to the different target coronary arteries was performed, see table 3 . The difference in patency between on- and off-pump was $6.5 \%$ (95\% CI -7.1 - 20.1; $\mathrm{p}=0.35$ ), i.e. superiority was not found. For the target coronary arteries, statistically significant differences were found for $\mathrm{LAD} / \mathrm{D}$ vs $\mathrm{Cx}$ (difference $10.6,95 \% \mathrm{CI} 2.0-19.2 ; \mathrm{p}=0.02$ ), LAD/D vs posterior descending artery (PDA) (difference 26.2, 95\% CI $10.4-41.9 ; \mathrm{p}<0.001$ ) and LAD/D vs Cx or PDA (difference 17.9, 95\% CI $9.2-26.6 ; \mathrm{p}<0.001$ ). The difference in patency between Cx and PDA was not significant, 15.6 (95\% CI $-2.2-33.4 ; \mathrm{p}=0.09)$.

As superiority for either of the two surgical procedures was not found, we analyzed a possible equivalence between graft patency in the on- and off-pump groups, see figure 3. We tested an equivalence with a span of at most +- 10 percentage units. As indicated in the figure, the upper $95 \%$ CI limit exceeds the limit of $10 \%$ units (15.79) and the lower 95\% CI limit is below zero, reaching the value of -2.78 . In accordance with Fleming ${ }^{11}$ and Blackwelder ${ }^{12}$ this indicates an inconclusive result, thus neither superiority nor equivalence can be statistically verified, and this is most likely due to a lack of power.

\section{Discussion}

Exceptional results of the NT SVGs have been demonstrated when used in on-pump CABG ${ }^{3-5}$. To our knowledge, no randomized study on patency rates of NT SVGs in off-pump CABG has been reported. This is a sub-study of a large international randomized multicenter trial ${ }^{9}$ that was designed to compare the clinical outcomes in high risk patients operated with either on- or off-pump CABG. Graft patency of the conduits was not investigated in this study. As we routinely use NT SVGs at our department, all of our patients included in this trial received one or two NT SVGs in addition to the LITA graft. Thus, the aim of this study was to evaluate the patency of the NT SVGs in both on- and off-pump CABG.

There was no superiority of on-pump CABG in terms of graft patency, $91.3 \%$ vs $84.7 \%$ for on- and off-pump respectively, $\mathrm{p}=0.35$. The patency was excellent at five years for both the LITA and NT SV grafts when used to bypass the LAD territory, 29/30 (96.7\%) for the LITA vs 32/32 (100\%) for NT SVGs independent of the surgical technique used. This is in agreement with our previous results ${ }^{13}$. On the other hand, the patency rates of NT SVGs to the right coronary targets were clearly less favorable, $80 \%$ in on-pump vs $62.5 \%$ in offpump surgery. Further analysis showed that $24.7 \%$ of the PDAs in this study had a diameter around $1 \mathrm{~mm}$, potentially jeopardizing the patency of SVGs attached to these vessels. These results differed from earlier results of a randomized trial with on-pump CABG in which the patency rate of NT SVGs anastomosed to the PDA at 1.5, 8.5 and 16 years were $100 \%, 95 \%$ and $94 \%$ respectively ${ }^{3-5}$.

The ROOBY and DOORS trials ${ }^{14,15}$ reported similar results with lower patency of grafts used to bypass the right coronary region in off-pump CABG. ROOBY showed $75.6 \%$ patency at one year while DOORS reported $72 \%$ patency at six months postoperatively. This was also true for both SV and arterial grafts used in a composite formation to bypass the right coronary artery (RCA). Hwang et al. reported lower patency of SV and arterial grafts to the RCA at one year postoperatively, although the patency was similar between the different conduits, $84.9 \%$ vs 82.9 for SV and arterial grafts respectively ${ }^{16}$. There are several 
possible explanations for this discrepancy in the patency rates of grafts anastomosed to different coronary territories between on- and off-pump CABG. Difficulties in surgical access, a demanding technique requiring more surgical experience and smaller target vessels could be the most acceptable justifications.

Kim et al. reported improved NT SVG patency in off-pump CABG in the context of composite grafts one year postoperatively ${ }^{17}$. The patency rate in the NT group was significantly higher than the minimal manipulation SVG group before and after propensity score matching, before, $97.4 \%$ vs $92.4 \%, p=0.024$; after, $97.3 \%$ vs $92.6 \%, p=0.051$. Hence, the NT SVG may improve the results and simplify a complex off-pump procedure especially when it comes to sequential grafts where the surrounding tissue in NT SVGs remains intact and acts as a biological, external stent protecting the long sequential grafts from kinking and reducing the potential for technical error. The number of grafts and distal anastomoses was higher in onpump than in the off-pump group (3.8 vs 3.1). This corresponds with several previous studies ${ }^{18} 19$. Although previous studies have shown no differences in graft patency between hand-sewn proximal anastomoses with the clamp-less Heartstring device vs partial clamp ${ }^{20}$, we chose to use the clamp-less technique avoiding excessive manipulation of the aorta.

The NT SVG was used to substitute the LITA in low-grade stenosis, in cases where the LITA was surgically damaged, and in patients with multiple co-morbidities. This is in accordance with a previous study on a similar group of patients. Hence, the patency rate of NT SVGs to the LAD was $98 \%$ at a meantime of six years ${ }^{13}$. The patients who received a planned SVG to the LAD were older, hade a lower left ventricular ejection fraction, higher risk scores, a low grade stenosis in the LAD and underwent concomitant procedures. The obvious limitation of our study is the small number of patients. Being a sub-study, the power estimation was not calculated to investigate the current issue.

\section{Acknowledgement}

We wish to express our gratitude to the nurses and staff at the Department of Cardiothoracic and Vascular Surgery and the Department of Radiology, Orebro University Hospital. Special thanks to the research staff; Kerstin Bergstrom, Catarina Gerdevag, Lenore Haugland and Lennart Johannesson for their thorough collection of data and kind care of the patients.

\section{Conclusion}

Comparable five-year patency rate for the NT SVG and the LITA to the LAD territory in both on- and off-pump CABG. Saphenous vein graft patency is lower to the right coronary artery with both surgical techniques but especially with off-pump CABG.

Table 1. Patient characteristics at five-year follow-up (baseline data reported at the end of the table).

$\mathrm{ACE}=$ angiotensin converting enzyme; $\mathrm{ASA}=$ acetyl salicylic acid; $\mathrm{CABG}=$ coronary artery bypass grafting; $\mathrm{CA}=$ calcium; $\mathrm{CCS}=$ Canadian Cardiovascular Society; IHD=ischemic heart disease; NYHA=New York Heart Association; $\mathrm{PCI}=$ percutaneous coronary intervention; $\mathrm{SD}=$ standard deviation.

Table 2. Distribution of conduit patency by number of distal anastomoses and target coronary arteries.

LITA=left internal thoracic artery; $\mathrm{SVG}=$ saphenous vein graft.

Table 3. Difference in graft patency according to distal anastomoses between on- and off-pump coronary artery bypass grafting stratified to the different coronary territories.

$\mathrm{CI}=$ confidence interval; $\mathrm{Cx}=$ circumflex; $\mathrm{D}=$ diagonal; $\mathrm{PDA}=$ posterior descending artery.

Figure 1. Flow chart of the baseline randomization and inclusion at five-year follow-up.

$\mathrm{CTA}=$ computed tomography angiography; distal=distal anastomoses; LITA=left internal thoracic artery; $\mathrm{n}=$ number; $\mathrm{SVG}=$ saphenous vein graft. 
Figure 2. Total patency (left internal thoracic artery and no-touch saphenous vein grafts) according to the coronary artery targets.

$\mathrm{DA}=$ diagonal artery; $\mathrm{LAD}=$ left anterior descending artery; $\mathrm{MA}=$ marginal artery; $\mathrm{RCA}=$ right coronary artery.

Figure 3. Total graft patency in the on- and off-pump groups tested with respect to potential equivalence and superiority between the groups, see text.

$\mathrm{CI}=$ confidence interval.

\section{References}

1. Caliskan E, de Souza DR, Boning A, Liakopoulos OJ, Choi YH, Pepper J, et al. Saphenous vein grafts in contemporary coronary artery bypass graft surgery. Nat Rev Cardiol 2020;17(3):155-69.

2. Souza D. A new no-touch preparation technique. Technical notes. Scand J Thorac Cardiovasc Surg 1996;30(1):41-4.

3. Souza DSR, Dashwood MR, Tsui JCS, Filbey D, Bodin L, Johansson B, et al. Improved patency in vein grafts harvested with surrounding tissue: Results of a randomized study using three harvesting techniques. Ann Thorac Surg 2002;73(4):1189-95.

4. Souza DS, Johansson B, Bojo L, Karlsson R, Geijer H, Filbey D, et al. Harvesting the saphenous vein with surrounding tissue for CABG provides long-term graft patency comparable to the left internal thoracic artery: results of a randomized longitudinal trial. J Thorac Cardiovasc Surg 2006;132(2):373-8.

5. Samano N, Geijer H, Liden M, Fremes S, Bodin L, Souza D. The no-touch saphenous vein for coronary artery bypass grafting maintains a patency, after 16 years, comparable to the left internal thoracic artery: A randomized trial. J Thorac Cardiovasc Surg 2015;150(4):880-8.

6. Lamy A, Devereaux PJ, Prabhakaran D, Taggart DP, Hu S, Paolasso E, et al. Off-pump or on-pump coronary-artery bypass grafting at 30 days. N Engl J Med 2012;366(16):1489-97.

7. Kowalewski M, Pawliszak W, Malvindi PG, Bokszanski MP, Perlinski D, Raffa GM, et al. Off-pump coronary artery bypass grafting improves short-term outcomes in high-risk patients compared with on-pump coronary artery bypass grafting: Meta-analysis. J Thorac Cardiovasc Surg 2016;151(1):60-77.e1-58.

8. Lamy A, Devereaux PJ, Prabhakaran D, Hu S, Piegas LS, Straka Z, et al. Rationale and design of the coronary artery bypass grafting surgery off or on pump revascularization study: a large international randomized trial in cardiac surgery. Am Heart J 2012;163(1):1-6.

9. Lamy A, Devereaux PJ, Prabhakaran D, Taggart DP, Hu S, Straka Z, et al. Five-Year Outcomes after Off-Pump or On-Pump Coronary-Artery Bypass Grafting. N Engl J Med 2016;375(24):2359-68.

10. Fitzgibbon GM, Kafka HP, Leach AJ, Keon WJ, Hooper GD, Burton JR. Coronary bypass graft fate and patient outcome: angiographic follow-up of 5,065 grafts related to survival and reoperation in 1,388 patients during 25 years. J Am Coll Cardiol 1996;28(3):616-26.

11. Fleming TR. Design and interpretation of equivalence trials. Am Heart J 2000;139(4):S171-6.

12. Blackwelder WC. "Proving the null hypothesis" in clinical trials. Control Clin Trials 1982;3(4):345-53.

13. Samano N, Geijer H, Bodin L, Arbeus M, Mannion JD, Dashwood M, et al. The no-touch saphenous vein graft in elderly coronary bypass patients with multiple comorbidities is a promising conduit to substitute the left internal thoracic artery. J Thorac Cardiovasc Surg 2017;154(2):457-66 e3.

14. Hattler B, Messenger JC, Shroyer AL, Collins JF, Haugen SJ, Garcia JA, et al. Off-Pump Coronary Artery Bypass Surgery Is Associated With Worse Arterial and Saphenous Vein Graft Patency and Less 
Effective Revascularization Results From the Veterans Affairs Randomized On/Off Bypass (ROOBY) Trial. Circulation 2012;125(23):2827-35.

15. Houlind K, Fenger-Gron M, Holme SJ, Kjeldsen BJ, Madsen SN, Rasmussen BS, et al. Graft patency after off-pump coronary artery bypass surgery is inferior even with identical heparinization protocols: Results from the Danish On-pump Versus Off-pump Randomization Study (DOORS). The Journal of Thoracic and Cardiovascular Surgery 2014;148(5):1812-9.e2.

16. Hwang HY, Kim JS, Kim K-B. Angiographic Equivalency of Off-Pump Saphenous Vein and Arterial Composite Grafts at One Year. The Annals of Thoracic Surgery 2010;90(2):516-21.

17. Kim YH, Oh HC, Choi JW, Hwang HY, Kim KB. No-Touch Saphenous Vein Harvesting May Improve Further the Patency of Saphenous Vein Composite Grafts: Early Outcomes and 1-Year Angiographic Results. Ann Thorac Surg 2017;103(5):1489-97.

18. Straka Z, Widimsky P, Jirasek K, Stros P, Votava J, Vanek T, et al. Off-pump versus on-pump coronary surgery: final results from a prospective randomized study PRAGUE-4. Ann Thorac Surg 2004;77(3):789-93.

19. Hu S, Zheng Z, Yuan X, Wang W, Song Y, Sun H, et al. Increasing long-term major vascular events and resource consumption in patients receiving off-pump coronary artery bypass: a single-center prospective observational study. Circulation 2010;121(16):1800-8.

20. Shimokawa T, Manabe S, Sawada T, Matsuyama S, Fukui T, Takanashi S. Intermediate-term patency of saphenous vein graft with a clampless hand-sewn proximal anastomosis device after off-pump coronary bypass grafting. Ann Thorac Surg 2009;87(5):1416-20.

\section{Hosted file}

Table 1.docx available at https://authorea.com/users/417762/articles/524745-five-yearpatency-for-the-no-touch-saphenous-vein-and-the-left-internal-thoracic-artery-in-onand-off-pump-coronary-artery-bypass-grafting

\section{Hosted file}

Table 2.docx available at https://authorea.com/users/417762/articles/524745-five-yearpatency-for-the-no-touch-saphenous-vein-and-the-left-internal-thoracic-artery-in-onand-off-pump-coronary-artery-bypass-grafting

\section{Hosted file}

Table 3.docx available at https://authorea.com/users/417762/articles/524745-five-yearpatency-for-the-no-touch-saphenous-vein-and-the-left-internal-thoracic-artery-in-onand-off-pump-coronary-artery-bypass-grafting 


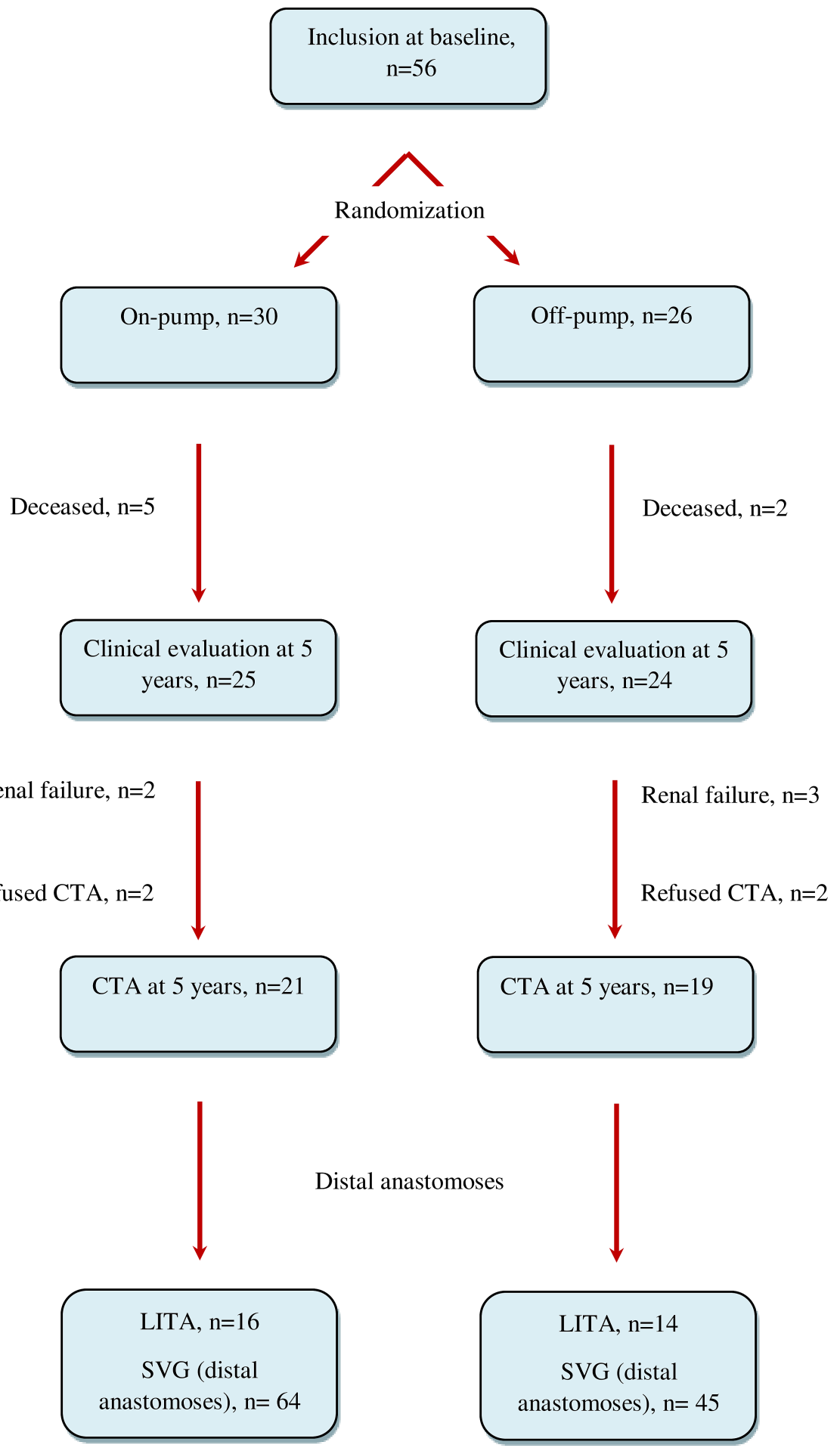



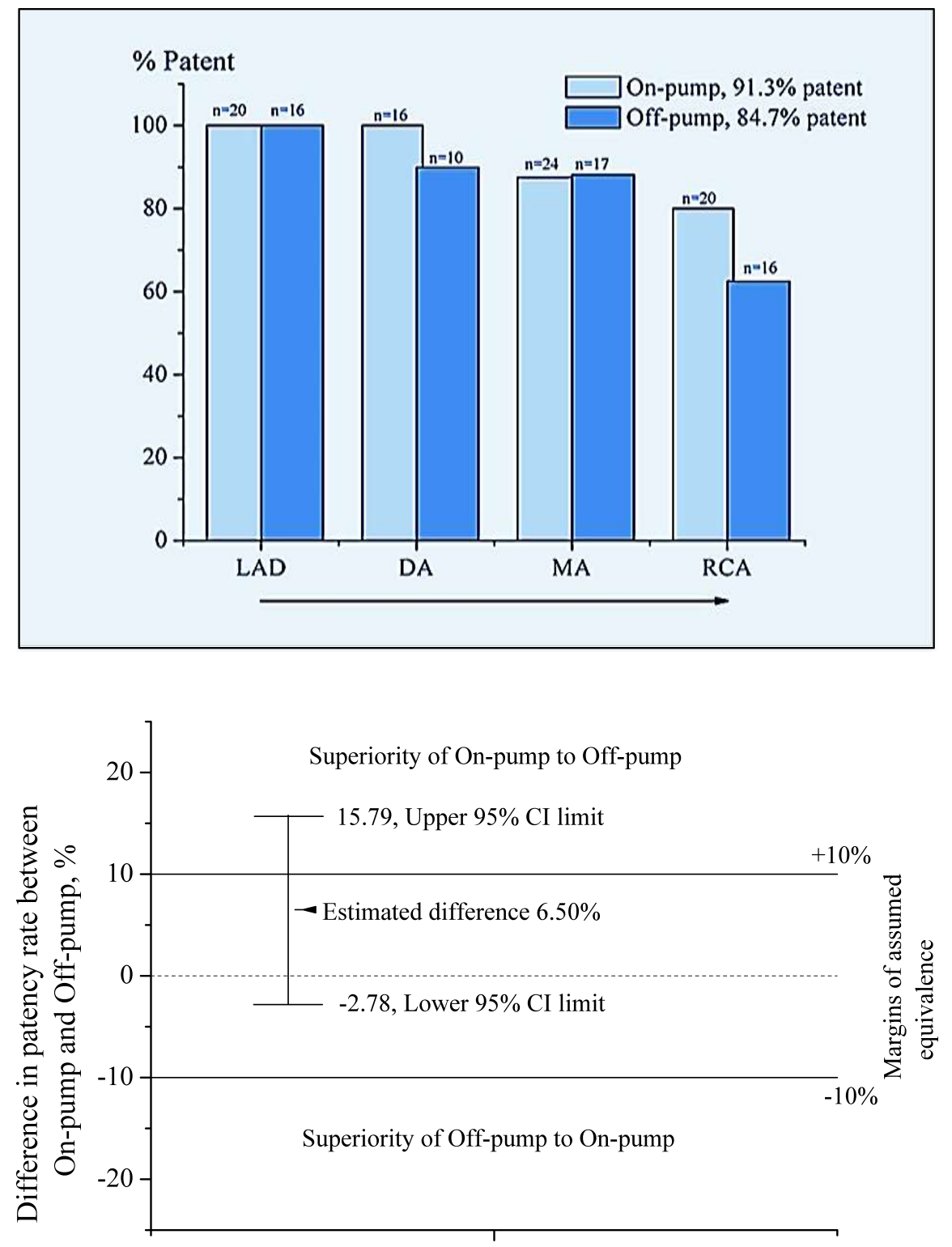